\title{
Superstructures of fluorescent cyclodextrin via click-reaction
}

\author{
Arkadius Maciollek ${ }^{1}$, Helmut Ritter ${ }^{* 1}$ and Rainer Beckert ${ }^{*}$
}

\section{Full Research Paper}

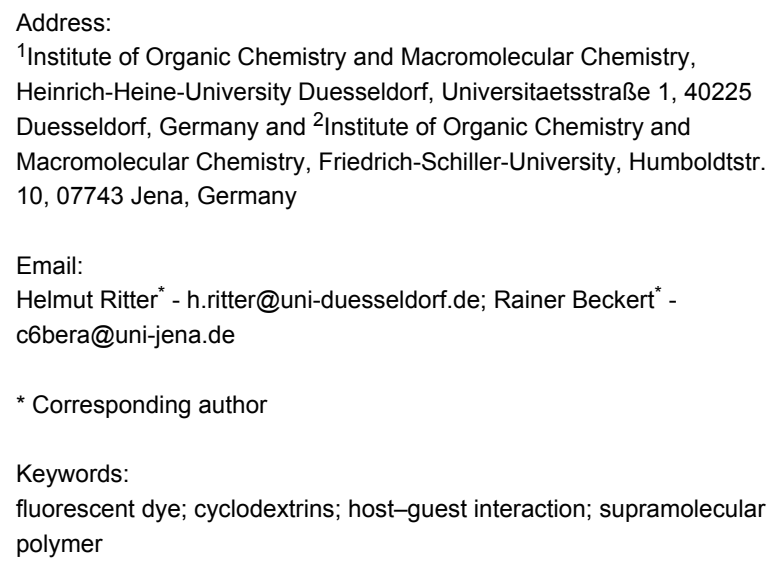

\section{Abstract}

Mono-(6-azido-6-deoxy)- $\beta$-cyclodextrin (CD) was covalently attached to an alkyne-modified 5-methyl-2-(pyridin-2-yl)thiazol-4-ol yielding a fluorophore containing $\mathrm{CD}$ in a click-type reaction. Intermolecular complexes were formed by poly(host-guest)-interactions. The supramolecular structures were characterized by ${ }^{1} \mathrm{H}$ NMR-ROESY spectroscopy, dynamic light scattering, UV-vis spectroscopy, fluorescence spectroscopy, and asymmetric flow field-flow fractionation. By adding potassium adamantane-1-carboxylate, the thiazol dye is displaced from the CD-cavity and the elongated noncovalent polymeric structures collapse.

\section{Introduction}

Most small heterocyclic molecules show low fluorescence. However, as we reported earlier, substituted 4-hydroxythiazoles have a high fluorescent nature $[1,2]$. These optical properties can be used for the development of sensor molecules, blue emitting dyes, luminescent materials or as donor- $(\pi$-conjugated-bridge)-acceptor-type dyes in dye-sensitized solar cells [3-5]. The etherification of 4-hydroxythiazole by using propargyl bromide opens up a wide field of modification through click chemistry with azides or thioles [6,7]. Accordingly, following our former work with mono azide modified $\mathrm{CD}$, we were encouraged to combine the fluorescent hydroxy-

thiazole dye with CD [8-10]. Such water soluble spectroscopically active hosts can be used as potentially biological markers, as molecular sensors, or for chiral recognition in aqueous solution [11-13]. Thus, in this present paper we describe the spectroscopic and structural behavior of a "CD-Click-Fluorophore" in the absence and presence of a competitive guest in water.

\section{Results and Discussion}

The water-soluble fluorescent cyclodextrin was synthesized via copper-catalyzed cycloaddition (Scheme 1). The existence of the resulting product was confirmed by MALDI-TOF spec- 


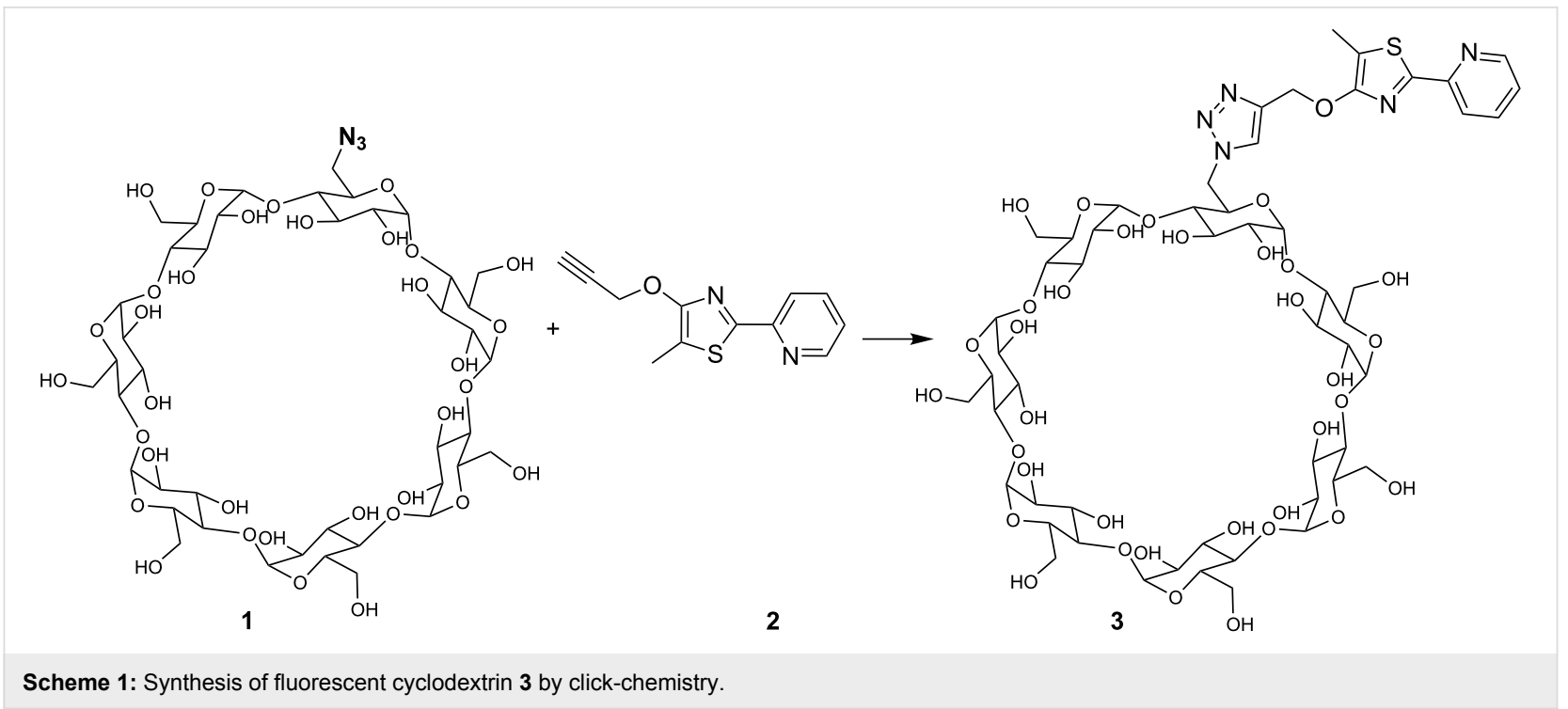

trometry, ${ }^{1} \mathrm{H}$ NMR and IR spectroscopy. The formation of host-guest structures between the thiazole functionality and $\beta$-CD was proven by ${ }^{1} \mathrm{H}$ NMR rotating-frame Overhauser effect spectroscopy (ROESY) (Figure 1). NOE correlation signals of the $\beta$-CD cavity protons between 4 and $3.5 \mathrm{ppm}$ and the aromatic protons of the pyridine moiety $8.6-7.4 \mathrm{ppm}$ were observed, clearly proving the formation of complexes.
However, no NOE interaction between protons of the methyl group of the thiazole and the triazole proton itself with $\mathrm{CD}$ is noticed. This indicates that only the inclusion of the pyridine moiety in the hydrophobic cavity of the CD takes place.

The formation of supramolecular structures was also proven by UV-vis spectroscopy and fluorescence spectroscopy. Figure 2

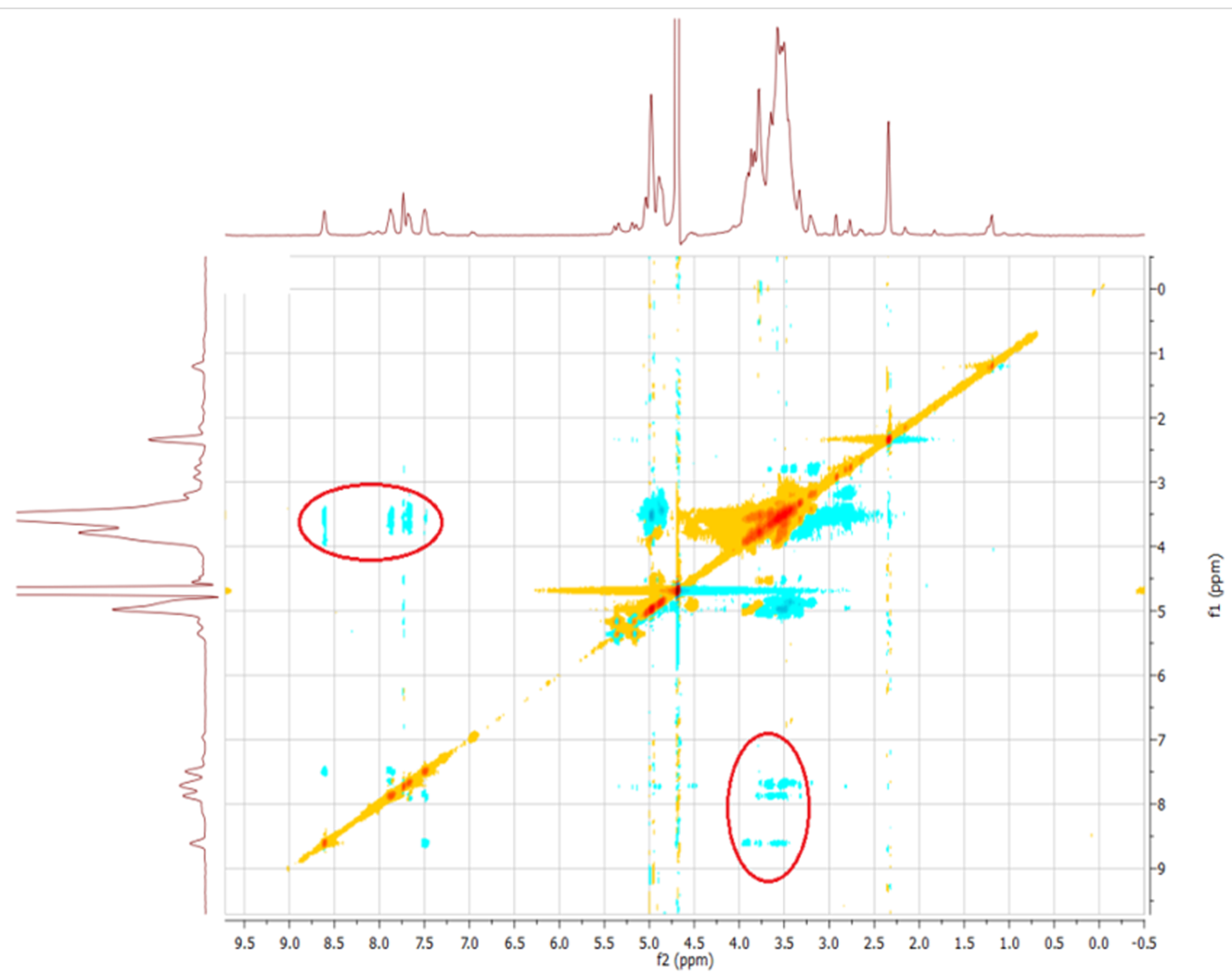

Figure 1: ${ }^{1} \mathrm{H}$ NMR-ROESY spectrum of the modified CD 3. 

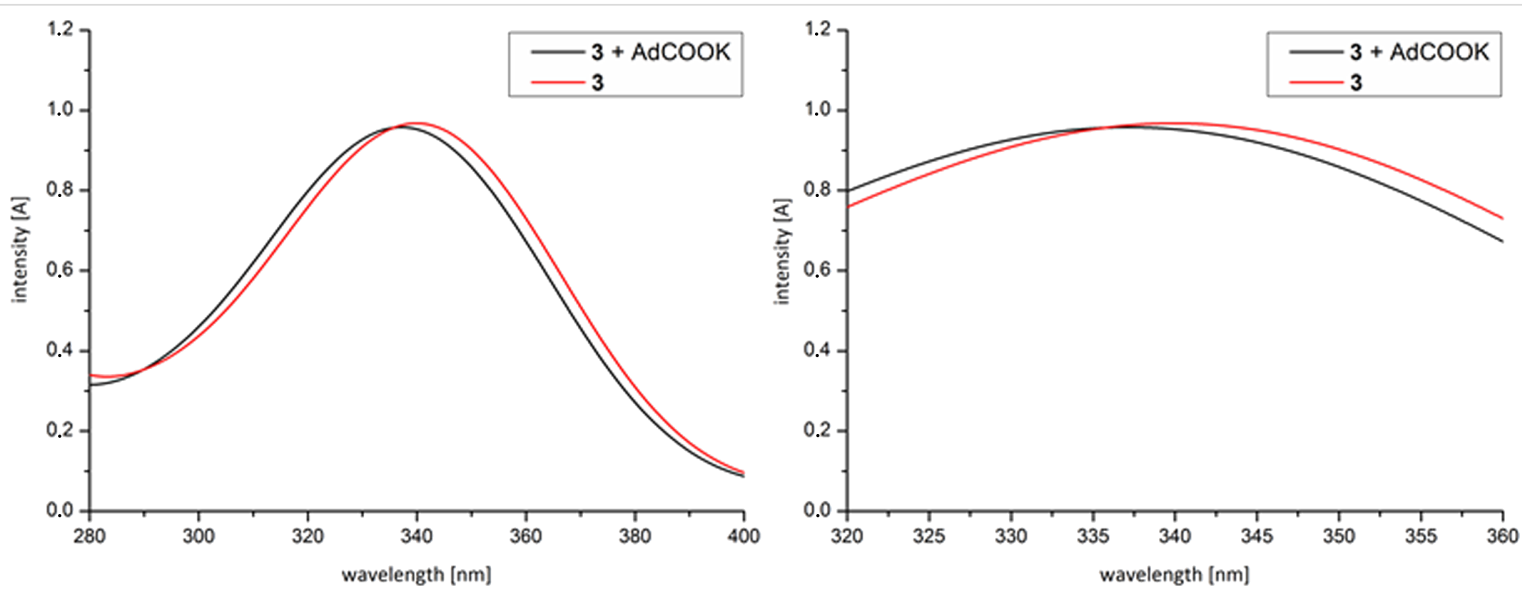

Figure 2: UV-vis spectrum of $3\left(4 \times 10^{-4} \mathrm{M}\right)$ with and without a 10 -fold excess of potassium adamantane-1-carboxylate.

shows the UV-vis absorption spectra of $\mathbf{3}$ in water with the characteristic band at $\lambda_{\max }=340 \mathrm{~nm}$. The addition of a 10 -fold excess of potassium adamantane-1-carboxylate, as competitive guest for CD leads to a hypsochromic shift with $\lambda_{\max }=336 \mathrm{~nm}$, through the exclusion of the fluorophore moiety from the hydrophobic cavity of $\mathrm{CD}$. This exchange causes a change in the HOMO-LUMO gap [14,15].

Figure 3 shows the fluorescence spectra of $\mathbf{3}$ in aqueous solution. Upon addition of potassium adamantane-1-carboxylate the maximum wavelength changes from 417 to $442 \mathrm{~nm}$ and the fluorescence intensity decreases. The inclusion of the fluorophore in the $\mathrm{CD}$ cavity causes an increase in the fluorescence intensity resulting from the decrease of the intramolecular rotational degrees of freedom of the molecule, compared to a solvent-accommodated fluorophore outside the cavity $[12,14,16]$.

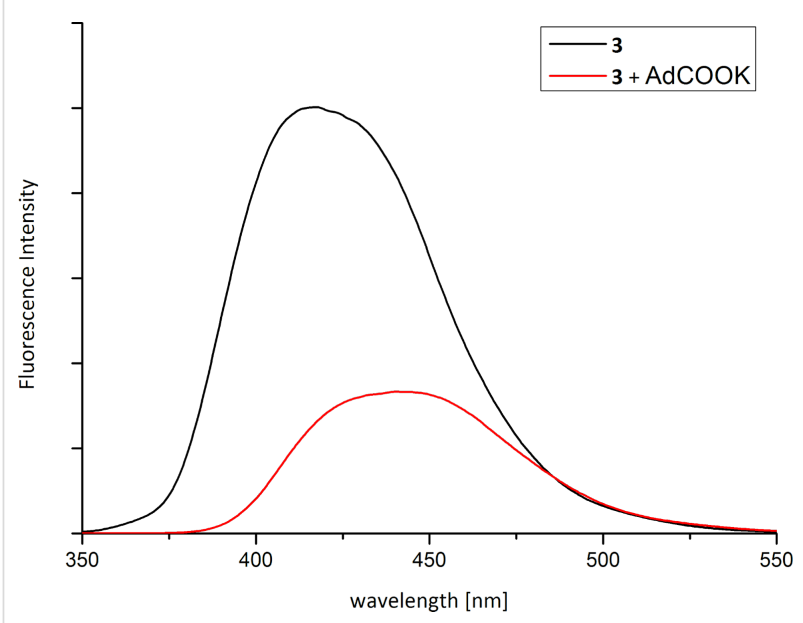

Figure 3: Fluorescence spectrum of $3\left(4 \times 10^{-4} \mathrm{M}\right)$ with and without a 10 -fold excess of 1 -adamantanecarboxylic acid
To investigate the expected intermolecular formation of supramolecular structures in aqueous solution, dynamic light scattering (DLS) experiments were performed (Figure 4). Hydrodynamic diameters up to $200 \mathrm{~nm}$ indicate the postulated formation of intermolecular complexes. Thus, repeating complexation of one rigid fluorophore moiety through the CD-function of the next monomer molecule takes place $[17,18]$. Accordingly, the formation of linear polymers via supramolecular monomer linkages can be claimed. Besides, intermolecular chain interactions may also take place due to hydrogen-bond interactions between the CD units.

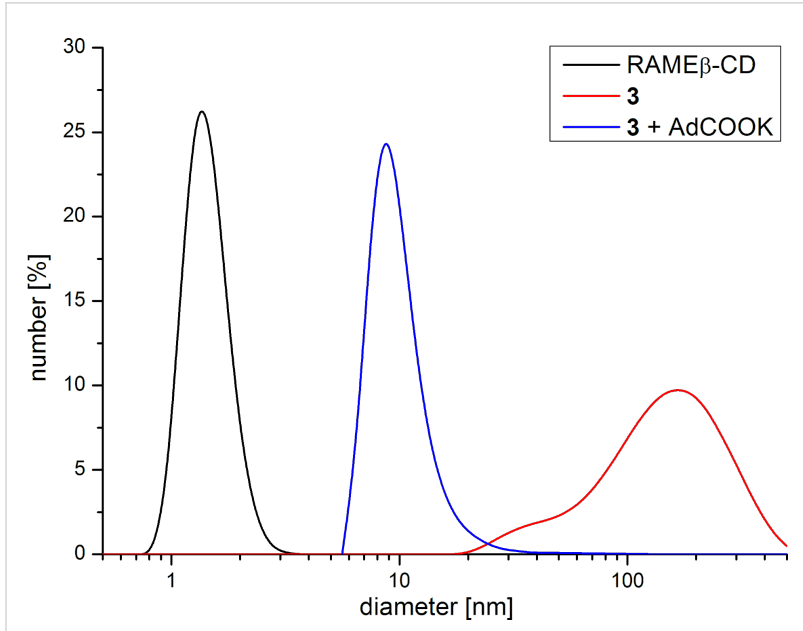

Figure 4: DLS measurement of $\mathbf{3}$ with and without a 10-fold excess of potassium adamantane-1-carboxylate; black: hydrodynamic diameter of randomly methylated $\beta-C D$, blue: mixture of monomer and oligomer after adding potassium adamantane-1-carboxylate, red: supramolecular polymer of 3 .

By adding potassium adamantane-1-carboxylate the pyridine moiety is displaced from the cavity. As a consequence, the noncovalent structures collapse to monomeric and oligomeric 
$\mathrm{CD}$ units and the hydrodynamic diameter decreases by as much as $5 \mathrm{~nm}$. Asymmetric flow field-flow fractionation experiments coupled with a three-angle light scattering detector confirm also the formation of intermolecular superstructures (Figure 5). Structures with a molar mass of $M_{\mathrm{n}}$ up to $100,000 \mathrm{~g} / \mathrm{mol}$ and also a hydrodynamic diameter up to $200 \mathrm{~nm}$ were detected. The conformation plot, i.e., the plot of the $\log$ of the radius as a function of the $\log$ of the molar mass, with a value of 0.7 estimates an elongated shape of the noncovalent structures. Upon addition of potassium adamantane-1-carboxylate no signals of the polymeric structure were detected.

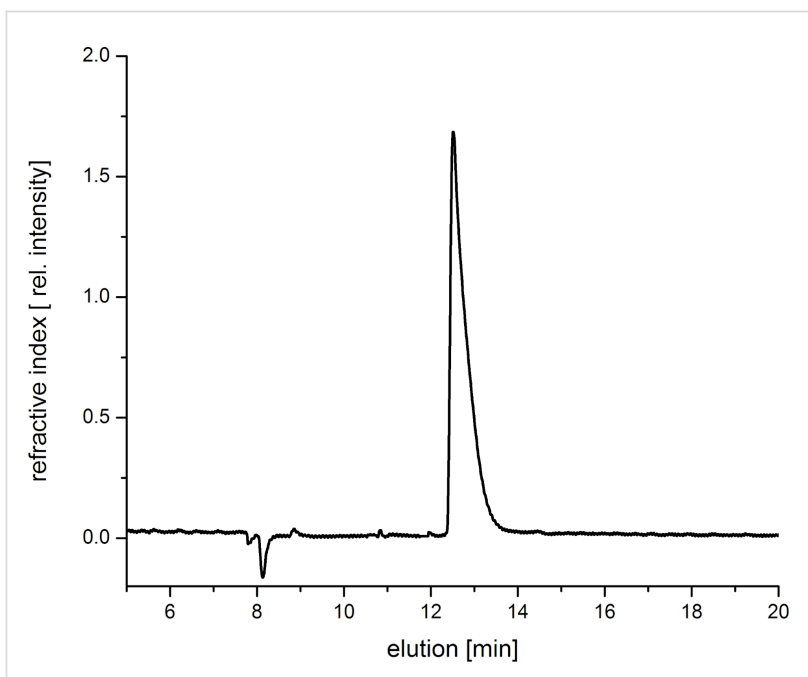

Figure 5: AF4 elution diagram of 3.

\section{Conclusion}

In summary, we have presented the synthesis of a fluorescent cyclodextrin via click reaction. The changes in the spectroscopic properties of the fluorescent cycloadduct were investigated in the presence and absence of the competitive guest potassium adamantane-1-carboxylate. The intermolecular formation of polymeric structures with elongated shape, through poly(host-guest)-interactions, was found. Furthermore, we were able to show the collapse of the supramolecular polymers upon addition of potassium adamantane-1-carboxylate.

\section{Experimental \\ General remarks}

All reagents used were commercially available (Sigma-Aldrich, Acros Organics) and used without further purification. $\beta-C D$ was obtained from Wacker Chemie GmbH, Burghausen, Germany and was used after drying overnight with a vacuum oil pump over $\mathrm{P}_{4} \mathrm{O}_{10} . \mathrm{N}, \mathrm{N}$-Dimethylformamide (DMF) was purchased from Fluka, Germany. Dimethyl sulfoxide- $d_{6}$ (99.9 atom \% D) and deuterium oxide, $\mathrm{D}_{2} \mathrm{O}$, were obtained from Deutero GmbH, Germany. ${ }^{1} \mathrm{H}$ NMR spectra were recorded on a Bruker Avance DRX 300 at $20^{\circ} \mathrm{C}$, shifts $(\delta)$ are given relative to signals arising from the solvent.

FT IR spectra were recorded on a Nicolet 6700 FT IR spectrometer equipped with an ATR unit. Matrix-assisted laser desorption/ionization-time-of-flight mass spectrometry (MALDI-TOFMS) was performed on a Bruker Ultraflex TOF mass spectrometer. Ions were formed with a pulsed nitrogen laser $(25 \mathrm{~Hz}, 337 \mathrm{~nm})$ and the molecular masses were recorded in linear mode. 2,5-Dihydroxybenzoic acid (DBH) in acetonitrile/water was used as a matrix.

Mass spectrometric experiments (MS) were performed on a Thermo Finnigan Trace DSQ (Dual-Stage Quadrupole) mass spectrometer. Ionization was carried out by electron ionization (EI). The absorption spectra were measured on a Specord 210 Plus UV-visible spectrophotometer. Fluorescence spectra were recorded on a Perkin Elmer LS55 luminescence spectrometer. AF4 measurements in ultrapure water were carried out on a combined system comprising the following elements: refractive index detector Optilabrex (Wyatt Technologies, laser wavelength $658 \mathrm{~nm}$ ), three angle light scattering detector miniDawn TREOS (Wyatt Technologies, laser wavelength $658 \mathrm{~nm}$, detector angles at $43.5^{\circ}, 90.0^{\circ}$ and $136.5^{\circ}$ ), UV detector Waters 486 (Waters), pump, degasser and autosampler (Agilent 1200, Agilent technologies). The molecular weight was calculated with Astra5 software from static light scattering data, by using the Zimm model. As concentration source, the refractive index was used. Calibration of the system was performed with bovine serum albumin. Dynamic light scattering (DLS) experiments were carried out with a Malvern Zetasizer Nano; ZS ZEN 3600 at a temperature of $20{ }^{\circ} \mathrm{C}$. The particle size distribution is derived from a deconvolution of the measured intensity autocorrelation function of the sample by a General Purpose Method (non-negative least squares) algorithm included in the DTS software. Microwave-assisted synthesis was performed using a CEM Discover Synthesis Unit (monomode system). The temperature was measured by infrared detection maintained at a constant value by power modulation. Reactions were performed in closed vessels.

Synthesis of mono-(6-azido-6-deoxy)- $\beta$-CD (1): Mono-(6azido-6-desoxy)- $\beta$-cyclodextrin was synthesized according to the known procedure [8].

Synthesis of 2-[5-methyl-4-(prop-2-yn-1-yloxy)-1,3-thiazol2-yl]pyridine (2): The alkyne modified thiazol was synthesized according to the literature procedure [2].

Synthesis of fluorescent $\boldsymbol{\beta}$-cyclodextrin 3: $1.3 \mathrm{~g}$ (1.15 mmol) of mono-(6-azido-6-desoxy)- $\beta$-cyclodextrin (1), $396 \mathrm{mg}$ 
(1.73 mmol) 2-[5-methyl-4-(prop-2-yn-1-yloxy)-1,3-thiazol-2yl]pyridine (2), $29 \mathrm{mg}(147 \mu \mathrm{mol})$ sodium ascorbate and $18 \mathrm{mg}$ (73.5 $\mu \mathrm{mol})$ copper(II) sulfate pentahydrate were suspended in $5 \mathrm{~mL}$ dimethylformamide in a pressure-resistant microwave test tube provided with a magnetic stirring bar. The tube was sealed and placed in the microwave and irradiated at $140{ }^{\circ} \mathrm{C}$ and $100 \mathrm{~W}$ for $30 \mathrm{~min}$. The product was precipitated in cold acetone, filtered and washed three times with acetone.

${ }^{1} \mathrm{H}$ NMR (300 MHz, DMSO- $\left.d_{6}, \mathrm{rt}\right) \delta 8.57$ (br, $\left.1 \mathrm{H}, \mathrm{ArH}\right)$, 8.16-8.01 (br, 2H, CH, ArH), 7.98-7.86 (br, 1H, ArH), 7.47-7.38 (br, 1H, ArH), 5.79-5.68 (br, 14H, OH), 5.40 (br, $\left.2 \mathrm{H}, \mathrm{CH}_{2}\right), 4.89-4.79$ (br, 7H, CH), 4.51-4.44 (br, 6H, OH), 3.75-3.53 (br, 28H, CH), $2.22\left(\mathrm{br}, 3 \mathrm{H}, \mathrm{CH}_{3}\right) \mathrm{ppm}$; IR: $3301(\mathrm{OH}), 2923(\mathrm{C}-\mathrm{H}), 1653(\mathrm{C}=\mathrm{C}), 1548$ (ring vibr.), $1329(\mathrm{OH}), 1153(\mathrm{COH}), 1078$ (COC), $1027(\mathrm{COH}) \mathrm{cm}^{-1}$; MS (MALDI-TOF) (acetonitrile/water 1:10): $m / z=1412[\mathrm{M}+\mathrm{Na}]^{+}$.

\section{References}

1. Grummt, U.-W.; Weiss, D.; Birckner, E.; Beckert, R. J. Phys. Chem. A 2007, 111, 1104-1110. doi:10.1021/jp0672003

2. Täuscher, E.; Weiß, D.; Beckert, R.; Görls, H. Synthesis 2010, 1603-1608. doi:10.1055/s-0029-1219759

3. Menzel, R.; Täuscher, E.; Weiß, D.; Beckert, R.; Görls, H. Z. Anorg. Allg. Chem. 2010, 636, 1380-1385. doi:10.1002/zaac.200900523

4. Happ, B.; Schäfer, J.; Menzel, R.; Hager, M. D.; Winter, A.; Popp, J.; Beckert, R.; Dietzek, B.; Schubert, U. S. Macromolecules 2011, 44, 6277-6287. doi:10.1021/ma201193e

5. Menzel, R.; Ogermann, D.; Kupfer, S.; Weiß, D.; Görls, H.; Kleinermanns, K.; González, L.; Beckert, R. Dyes Pigm. 2012, 94 , 512-524. doi:10.1016/j.dyepig.2012.02.014

6. Lutz, J.-F. Angew. Chem. 2007, 119, 1036-1043. doi:10.1002/ange.200604050

7. Hoyle, C. E.; Lowe, A. B.; Bowman, C. N. Chem. Soc. Rev. 2010, 39, 1355-1387. doi:10.1039/b901979k

8. Choi, S.; Munteanu, M.; Ritter, H. J. Polym. Res. 2009, 16, 389-394. doi:10.1007/s10965-008-9240-0

9. Munteanu, M.; Choi, S.; Ritter, H. Macromolecules 2008, 41, 9619-9623. doi:10.1021/ma8018975

10. Maatz, G.; Maciollek, A.; Ritter, H. Beilstein J. Org. Chem. 2012, 8 , 1929-1935. doi:10.3762/bjoc.8.224

11. Ogoshi, T.; Harada, A. Sensors 2008, 8, 4961-4982. doi:10.3390/s8084961

12. Tanabe, T.; Touma, K.; Hamasaki, K.; Ueno, A. Anal. Chem. 2001, 73, 1877-1880. doi:10.1021/ac001062a

13. Ueno, A. Adv. Mater. 1993, 5, 132-134. doi:10.1002/adma.19930050213

14. Nowakowska, M.; Smoluch, M.; Sendor, D. J. Inclusion Phenom. Macrocyclic Chem. 2001, 40, 213-219. doi:10.1023/A:1011820513256

15. Hamasaki, K.; Ikeda, H.; Nakamura, A.; Ueno, A.; Toda, F.; Suzuki, I.; Osa, T. J. Am. Chem. Soc. 1993, 115, 5035-5040. doi:10.1021/ja00065a012
16. Ikeda, H.; Nakamura, M.; Ise, N.; Oguma, N.; Nakamura, A.; Ikeda, T.; Toda, F.; Ueno, A. J. Am. Chem. Soc. 1996, 118, 10980-10988. doi:10.1021/ja960183i

17. Harada, A. J. Polym. Sci., Part A: Polym. Chem. 2006, 44, 5113-5119. doi:10.1002/pola.21618

18. Munteanu, M.; Kolb, U.; Ritter, H. Macromol. Rapid Commun. 2010, 31, 616-618. doi:10.1002/marc.200900754

\section{License and Terms}

This is an Open Access article under the terms of the Creative Commons Attribution License

(http://creativecommons.org/licenses/by/2.0), which permits unrestricted use, distribution, and reproduction in any medium, provided the original work is properly cited.

The license is subject to the Beilstein Journal of Organic Chemistry terms and conditions:

(http://www.beilstein-journals.org/bjoc)

The definitive version of this article is the electronic one which can be found at: doi:10.3762/bjoc. 9.94 